\title{
Do Molecular Structures of Migraine Drugs Point to a Common Cause of this Elusive Disease and Suggest Future Drug Designs?
}

\author{
Mark J. Brinsden • Ian C. Shaw
}

Published online: 20 February 2015

(c) The Author(s) 2015. This article is published with open access at Springerlink.com

\section{Introduction}

We should, perhaps, consider migraine as a two-stage disorder. Stage 1 is the underlying biochemical disorder (migraine disorder) that predisposes the sufferer to stage 2, which is the manifestation of the disorder as a migraine headache.

The triggers of migraine headaches are multifarious. Some sufferers of migraine disorder link their migraine attacks to eating specific foods (e.g. chocolate, cheese), specific situations (e.g. stress), the time of year or even weather conditions [1].

Treatments for migraine are equally diverse and might be based on what are thought by the physician to be the underlying causes of the migraine attack (e.g. use of $\beta$-blockers to reduce local brain blood pressure) or use of drugs designed to treat the migraine headache per se (e.g. triptans).

\section{What is the Underlying Biochemistry of Migraine?}

There is no definitive answer to the question of what underlies migraine disorder or triggers migraine headaches. Current thinking points to migraine being a brain disorder related (at least in part) to serotonin [5-hydroxytryptamine (5-HT)] and/or dopamine (DA) and their receptors, and no single, simple, mechanism is thought to account for the disease [2]. When the actions elicited by DA are

M. J. Brinsden · I. C. Shaw $(\bowtie)$

Department of Chemistry, University of Canterbury, Private Bag 4800, Christchurch 8140, New Zealand

e-mail: ian.shaw@canterbury.ac.nz considered-including mind, mood and behaviour effects-the complexity of the disease is understandable.

The concentrations of 5-HT and DA in the synapse are important in determining their effects; for this reason, there are several synaptic concentration control mechanisms and several postsynaptic receptors that elicit different effects. The control mechanisms involve presynaptic 5-HT or DA receptors, which, when occupied, cause a series of cyclic adenosine monophosphate (cAMP)-mediated events that result in reduced release of either DA or 5-HT from presynaptic vesicles into the synapse. In addition, there are presynaptic membrane transporters for either DA (i.e. DAT) or 5-HT that pump the neurotransmitter from the synapse back into the presynaptic nerve terminal, where it is, in turn, pumped into presynaptic vesicles for reuse later. A combination of the DA or 5-HT transporters and the presynaptic feedback receptors together allow fine control of synaptic neurotransmitter concentrations (Fig. 1).

Both DA and 5-HT work by binding to postsynaptic receptors, resulting in initiation of a wave of depolarization (action potential), which elicits the response. In addition, similar receptors are found on muscles at neuromuscular synaptic junctions. Such junctions might exist between, for example, serotonergic nerves and blood vessel walls, resulting in vessel contraction and increased blood pressure. This might explain the link between migraine headaches, DA/5-HT and localized brain blood pressure changes [2].

Presynaptic inhibitory receptors are key facets of the control of neurotransmitter synthesis. Clearly, the relative binding constants for specific neurotransmitters (e.g. DA) to specific receptors determine the overall effect of a particular neurotransmitter concentration in the synapse. Therefore, the concentration of a particular neurotransmitter (or the ratio of concentrations between several neurotransmitters) in the synapse determines the overall effect. 


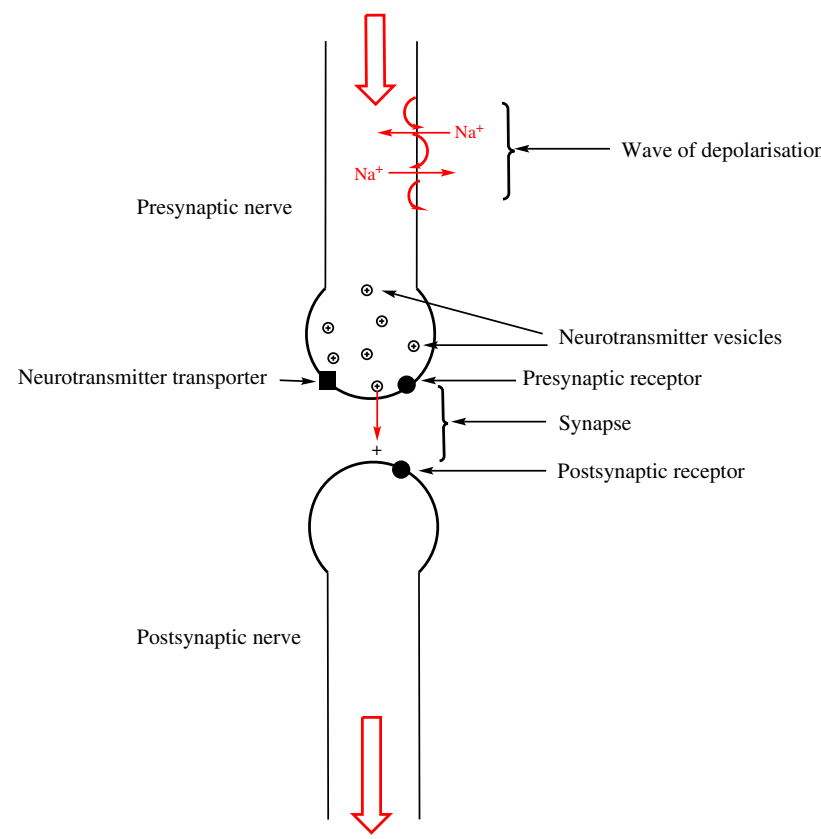

Fig. 1 Schematic representation of 5-hydroxytryptamine/dopamine (5-HT/DA)-mediated neurotransmission. Plus represents DA or 5-HT

Further to this, there is a plethora of receptor subtypes that have specific effects and binding constants for one or more neurotransmitters. For example, $5-\mathrm{HT}_{1 \mathrm{~B}}$ is a subtype of the 5-HT receptor family; 5-HT has various affinities for receptor subtypes, as do 5-HT mimics such as triptans (used to treat migraine headaches). In addition, there is a class of central nervous system (CNS) trace amine receptors (TAARs) that appear to be distinct from classical biogenic amine (e.g. 5-HT) receptors. TAARS bind tyramine, $\beta$-phenylethylamine, tryptamine and octopamine, which are present at trace levels in the mammalian CNS [3].

Trace amines (e.g. tyramine) have been implicated in the aetiology of migraine. Indeed, consumption of foods rich in tyramine (e.g. cheese) are often linked to headaches by migraine sufferers. Tyramine might be present in foods per se, it might be produced from tyrosine-rich foods by gut microfloral decarboxylation or it might be produced in the CNS from tyrosine. Tyramine is structurally analogous to 5-HT, and therefore it is possible that it interacts with 5-HT receptors or transporters, which might explain its link with headaches in some migraine sufferers. Interestingly, a clinical study carried out in the 1970s showed that administration of tyramine to migraine sufferers did not precipitate headaches [4]. This study focused on tyramine in isolation; however, it is possible that if tyramine has a role in migraine, it is more complex, and that dietary tyramine is only one component of a complex array of risk factors necessary to precipitate a headache. Many migraine sufferers still tell their doctors that their headaches are precipitated by tyramine-containing foods-even if Moffet and her colleagues argue otherwise [4].

From this brief discussion, it is clear that migraine disorder and/or migraine headache, their triggers and treatments are inextricably linked through possible interactions of 5-HT molecular mimics with postsynaptic receptors, presynaptic inhibitory receptors and/or transporters. The potential complexity of these interactions is overwhelming.

Migraine triggers might mimic neurotransmitters, leading to neurotransmitter synaptic concentration irregularities with consequent unscheduled nerve firing. This might explain phantom sensory effects, such as the flashing lights described by many migraine sufferers.

The functionality of this complex receptor/transporterbased control/effect system relies upon the differential binding affinities of the different receptors and transporters. These differential binding affinities ensure that the synaptic neurotransmitter concentrations determine occupancy of the postsynaptic receptors (and therefore the effect) and that above a particular concentration, binding to the presynaptic receptor occurs (thus inhibiting neurotransmitter synthesis) and/or that binding to the transporter occurs (thus reducing synaptic neurotransmitter concentration).

We have focused on DA and 5-HT thus far; however, other neurotransmitters operate by similar systems (e.g. noradrenaline) and have also been implicated in the aetiology of migraine [5]. This adds further to the complexity of this receptor-mediated disease.

It is clear that if a molecule or a stimulus that releases a molecule that interferes with any aspect of this system occurs that profound neurological effects might result. For example, tyramine present in chocolate, cheese and soy sauce has structural analogies with DA (and noradrenaline) [Fig. 2] and so could interact with the dopaminergic system by binding to DA receptors or DAT, which might explain its link to migraine in some people [4].

Interestingly, migraine disorder is more common in women than men [6], is rare in children, is relieved in
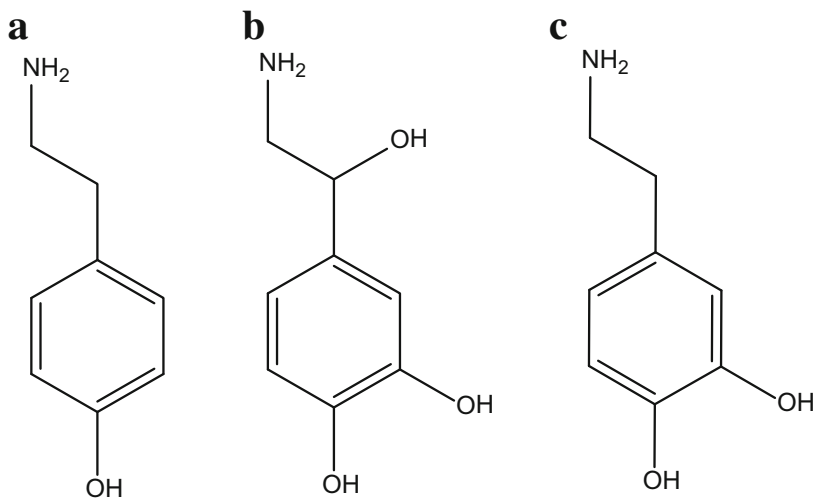

Fig. 2 a Tyramine. b Noradrenaline. c Dopamine 
pregnant migraine sufferers in the third trimester [7], transdermal estrogens can be used in prophylactic treatment, and it is rare in postmenopausal women. Clearly, estrogen(s) is/are likely involved in the disorder. Interestingly, $17 \beta$-estradiol (E2) modulates $5-\mathrm{HT}_{1}$ receptors in the rat brain [8], which suggests a link between E2 levels and 5-HT-mediated effects.

\section{Treatment of Migraine}

Treatment can be classified as either acute or preventative [9-11]; acute treatment is further divided into migrainespecific or non-specific treatment [11]. The former ostensibly impacts on the cellular mechanisms of the disease (e.g. local cerebral blood pressure changes), while the latter focuses on underlying migraine triggers (e.g. anxiety).

Specific migraine treatments include ergotamine, dihydroergotamine (DHE) and triptans (e.g. rizatriptan). These drugs are all agonists of 5-HT receptors; ergotamine is a $5-\mathrm{HT}_{1 \mathrm{~B}}$ receptor agonist, $\mathrm{DHE}$ is a $5-\mathrm{HT}_{1}$ receptor agonist [12] and the triptans are 5- $\mathrm{HT}_{1 \mathrm{~B} / 1 \mathrm{D}}$ agonists [11]. Such drugs rapidly ameliorate the migraine headache, presumably by addressing anomalies in synaptic 5-HT concentrations.

There are many preventative drugs given to reduce the frequency and severity of anticipated migraines [13]; their effects and efficacies are variable [10]. These treatments can be classified according to their primary therapeutic uses, including $\beta$-blockers (e.g. propranolol), antidepressants (e.g. amitriptyline), calcium channel antagonists (e.g. verapamil), anticonvulsants (e.g. valproic acid) and 5-HT receptor antagonists (e.g. pizotifen) [14].

It is interesting that despite the preventative drugs having very different primary therapeutic uses, they (or their metabolites) have molecular similarities and significant structural analogies with DA or 5-HT (Fig. 3); therefore, it is possible that these drugs, despite the therapeutic rationale underlying their efficacy in migraine treatment, work via mechanisms related to DA and/or 5-HT receptor or transporter systems, which might not be their primary modes of action. On the other hand, the primary modes of action of some of these drugs might indeed be via DA or 5-HT receptor interactions, but the desired outcome was to treat other DA/5-HT-related disorders (e.g. depression) for which the drugs were originally 'designed', rather than treating migraine. In these cases, the drugs' usefulness in migraine treatment is fortuitous.

The structural analogies shown in Fig. 3 are based on either major metabolite or parent molecule structural similarities with neurotransmitters. A closer look at the metabolic pathways of amitriptyline and verapamil reveals other metabolites with closer structural analogies to the a<smiles>CC(C)NCCCOc1ccc(O)c2ccccc12</smiles>

b
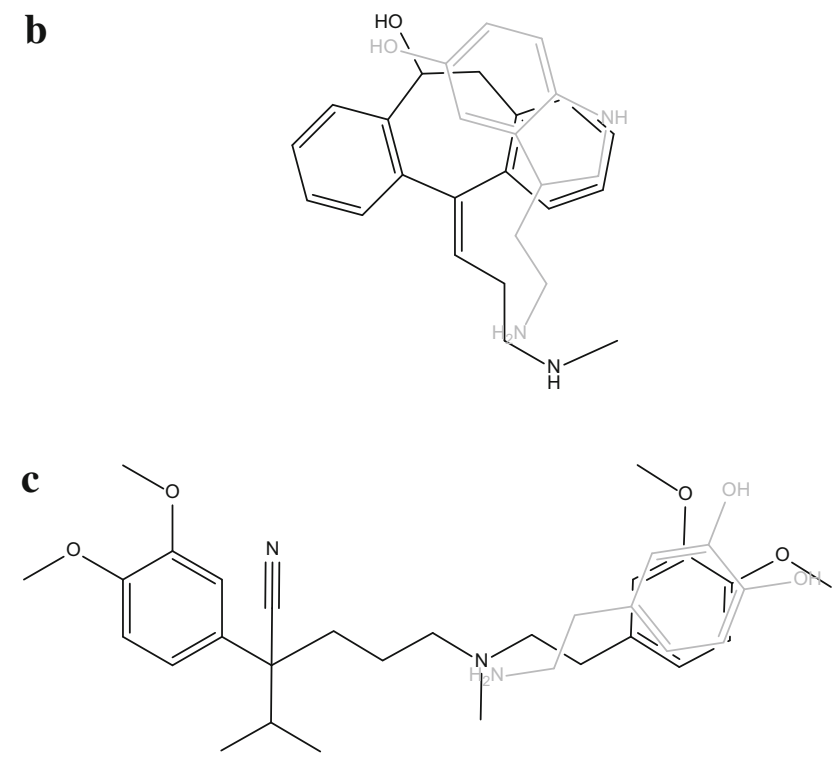

Fig. 3 Molecular structures of a the hydroxy-metabolite of the $\beta$-blocker propranolol [black], showing its structural analogy to dopamine (DA) [grey]; $\mathbf{b}$ the hydroxy-metabolite of the antidepressant amitriptyline [black], showing its structural analogy to 5-hydroxytryptamine (5-HT) [ grey]; and $\mathbf{c}$ the antianginal antiarrhythmic verapamil [black], showing its structural analogy to DA [grey]

neurotransmitters. For example, verapamil is metabolized by cleavage of the $\mathrm{C}-\mathrm{N}$ bond in the aliphatic chain that links its two aromatic centres (see Fig. 3) to form a metabolite (M6) [15], which has a significant threedimensional structural analogy to DA (Fig. 4).

Interestingly, mood and feeling of wellbeing are affected by DA and or 5-HT [16], which likely explains the modes of action of tricyclic antidepressants [17] (e.g. amitriptyline and imipramine) and, in turn, might explain why these drugs are also useful in the treatment of migraine. Indeed, pizotifen, a drug primarily used for migraine treatment, has structural analogies with 5-HT and is a 5-HT antagonist [18].

A closer look at the molecular similarities between drugs used to treat migraine, their molecular analogies with DA or 5-HT and their possible interactions with, for example, 5-HT receptors might give an insight into the molecular structures for new antimigraine drugs. It might even be possible to specify molecular dimensions in terms of separation and orientation of key molecular moieties to achieve theoretical occupancy of receptors and/or 
Fig. 4 Three-dimensional representations of dopamine (DA) $[l e f t]$ and the verapamil metabolite M6 [right], seen from different orientations [above and below]. The molecular analogies are clear in a three-dimensional context, which supports the possibility that M6 could interact with the dopamine transporter at the DA binding site
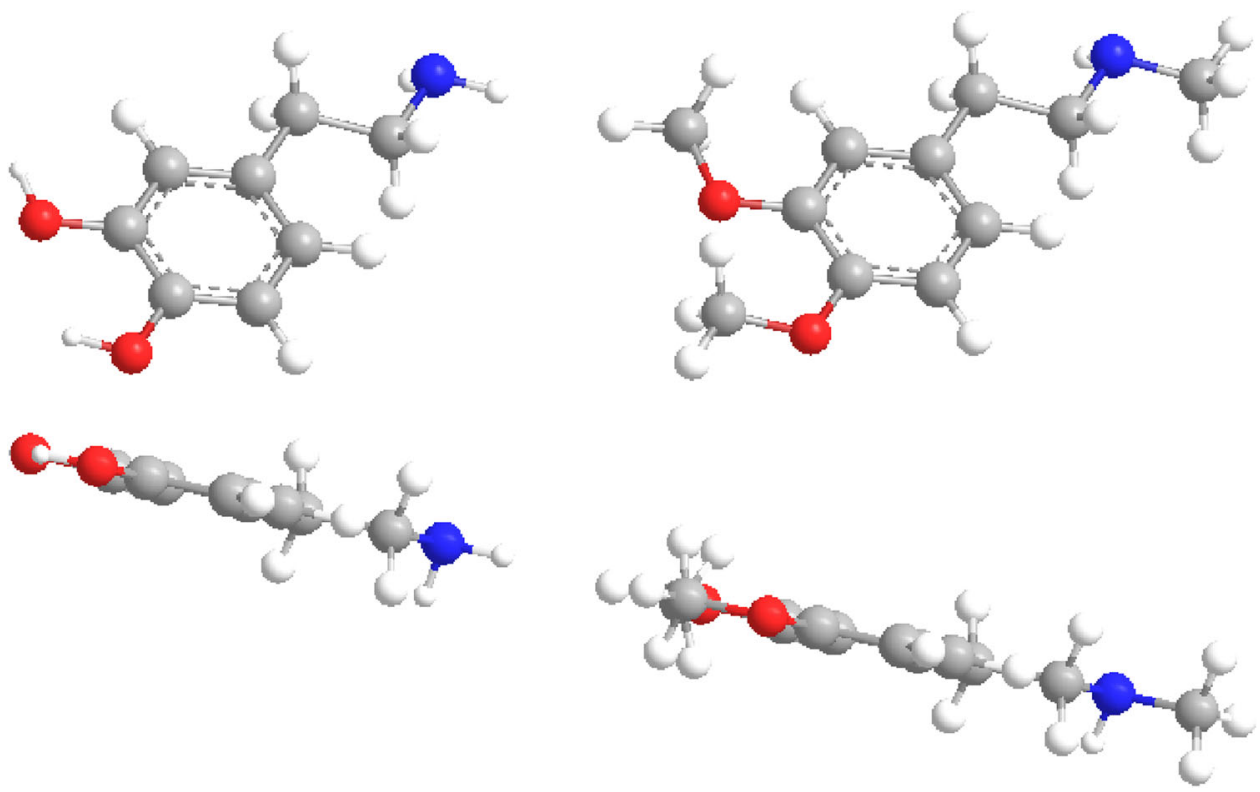

Fig. 5 5-Hydroxytryptamine (5-HT) imported in silico into a $5-\mathrm{HT}_{1 \mathrm{~B}}$-BRIL/ergotamine crystal structure [19] following removal of ergotamine, using ChemBio 3D Ultra, showing the hydrogen bonds (and their bond lengths), likely formed with key amino acid residues in the binding cleft

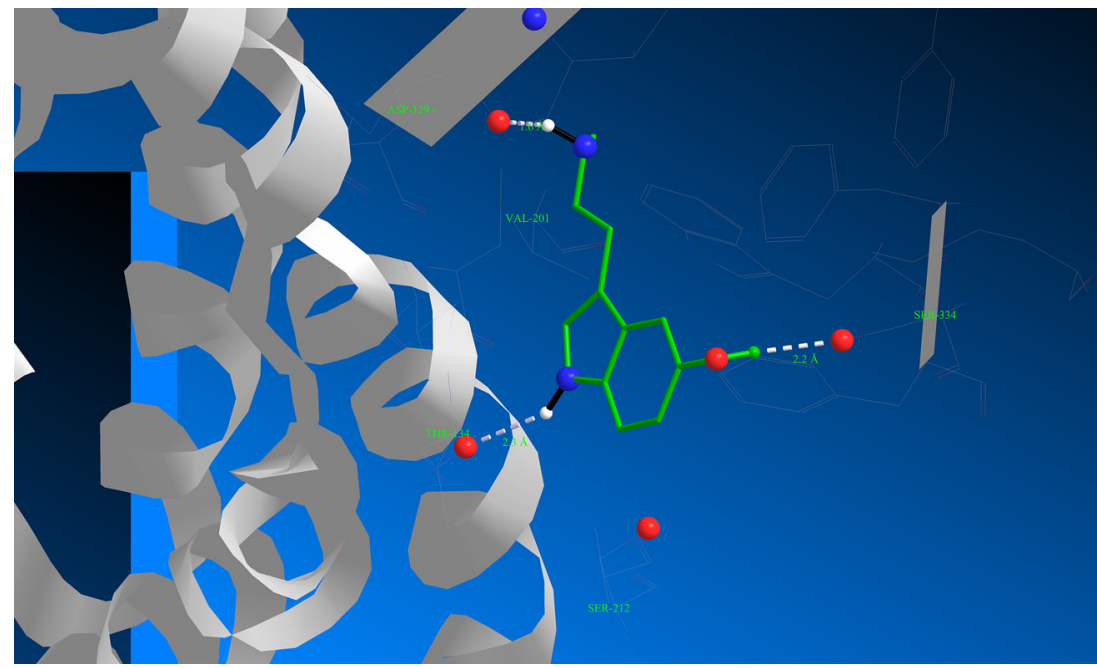

transporters. For example, importing 5-HT into the binding cleft of 5-HT receptor derived from a 5-HT $1 \mathrm{~B}$-BRIL/ergotamine crystal structure [19], using ChemBio 3D Ultra (Perkin Elmer), shows the best fit orientation of the ligand and the theoretical positions of hydrogen bonds with amino acid residues in the binding cleft (Fig. 5). The $5-\mathrm{HT}_{1 \mathrm{~B}}-\mathrm{BRIL/ergotamine}$ crystal structure is used for this example because there is no crystal structure published for a 5-HT receptor with 5-HT in the binding cleft. Ergotamine has been removed and 5-HT fitted so that it bonds to the same key amino acid residues as the agonist.

Hydroxyamitriptyline (OH-AT), a metabolite of amitriptyline, has a significant three-dimensional molecular structural analogy to 5-HT (Fig. 6). When OH-AT is superimposed onto 5-HT in ChemBio 3D, their side chain nitrogens are only $1.1 \AA$ apart and their ring hydroxyls are only $1.3 \AA$ apart. This suggests that $\mathrm{OH}-\mathrm{AT}$ would fit and hydrogen bond to $5-\mathrm{HT}_{1 \mathrm{~B}}$. In terms of molecular moiety separation, the ring hydroxyl and the side chain (or equivalent) nitrogen should be of the order of $5.6 \AA$ separated to fit and hydrogen bond to $5-\mathrm{HT}_{1 \mathrm{~B}}$. This is a simple example that illustrates the importance of threedimensional molecular dimensions and natural ligand mimicry in determining likely receptor fit and consequent potential pharmacological activity.

Therefore, it is likely that drugs used for the treatment of migraine were originally used because they were thought to treat the underlying cause of a particular patient's migraine. However, on further scrutiny, it is perhaps more likely that, fortuitously, these drugs have effects that also interact with the primary neurological mechanism of migraine. 


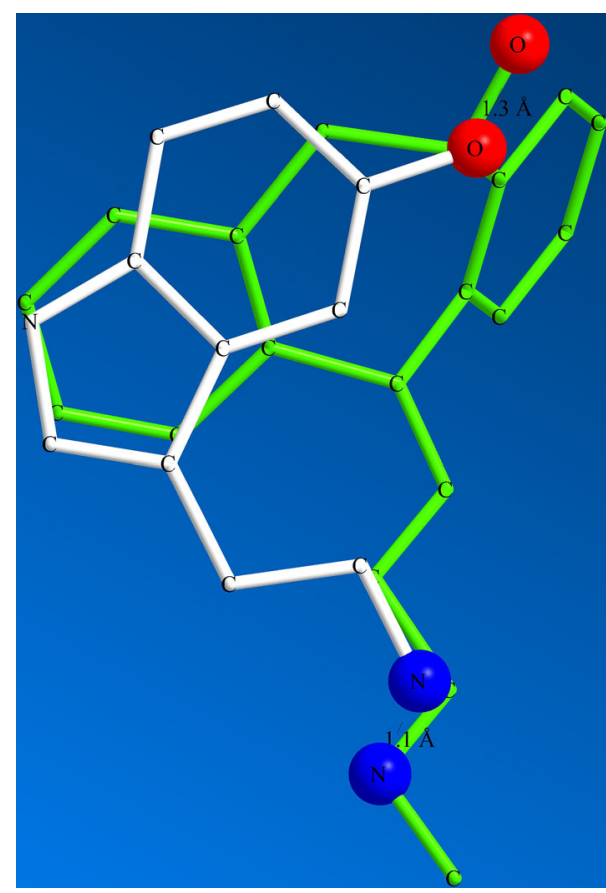

Fig. 6 Hydroxyamitryptyline (OH-AT), a metabolite of amitriptyline, superimposed on 5-hydroxytryptamine (5-HT), using ChemBio 3D, showing the close proximity of the side chain nitrogens and the ring hydroxyls, which suggests that OH-AT would occupy and interact with $5-\mathrm{HT}_{1 \mathrm{~B}}$ in a similar way to 5 -HT itself

This thinking not only supports the increasing evidence that migraine is a brain disorder involving the 5-HT and/or DA systems, but also provides a rationale for migraine drug design.

Acknowledgments We thank Dr Ben Hudson for helpful discussion on the treatment of migraine. No funding was received for this work. Mark Brinsden and Ian Shaw have no conflicts of interest

Open Access This article is distributed under the terms of the Creative Commons Attribution Noncommercial License which permits any noncommercial use, distribution, and reproduction in any medium, provided the original author(s) and the source are credited.

\section{References}

1. Wöber C, Holzhammer J, Zeitlhofer J, Wessely P, Wöber-Bingöl Ç. Trigger factors of migraine and tension-type headache: experience and knowledge of the patients. J Headache Pain. 2006;7(4):188-95.

2. Akerman S, Holland PR, Goadsby PJ. Diencephalic and brainstem mechanisms in migraine. Nat Rev Neurosci. 2011;12(10): 570-84.

3. Borowsky B, Adham N, Jones KA, Raddatz R, Artymyshyn R, Ogozalek KL, et al. Trace amines: identification of a family of mammalian G protein-coupled receptors. Proc Natl Acad Sci. 2001;98(16):8966-71.

4. Moffett A, Swash M, Scott D. Effect of tyramine in migraine: a double-blind study. J Neurol Neurosurg Psychiatry. 1972;35(4): 496-9.

5. Gotoh F, Komatsumoto S, Araki N, Gomi S. Noradrenergic nervous activity in migraine. Arch Neurol. 1984;41(9):951.

6. Stewart W, Wood C, Reed M, Roy J, Lipton R. Cumulative lifetime migraine incidence in women and men. Cephalalgia. 2008;28(11):1170-8.

7. Edelson RN. Menstrual migraine and other hormonal aspects of migraine. Headache. 1985;25(7):376-9.

8. Biegon A, McEwen B. Modulation by estradiol of serotonin receptors in brain. J Neurosci. 1982;2(2):199-205.

9. Silberstein SD. Treatment recommendations for migraine. Nat Clin Pract Neurol. 2008;4(9):482-9.

10. Snow V, Weiss K, Wall EM, Mottur-Pilson C. Pharmacologic management of acute attacks of migraine and prevention of migraine headache. Ann Intern Med. 2002;137(10):840-9.

11. Goadsby PJ, Lipton RB, Ferrari MD. Migraine-current understanding and treatment. N Engl J Med. 2002;346(4):257-70.

12. Pryse-Phillips WE, Dodick DW, Edmeads JG, Gawel MJ, Nelson RF, Purdy RA, et al. Guidelines for the diagnosis and management of migraine in clinical practice. Canadian Headache Society. Can Med Assoc J. 1997;156(9):1273-87.

13. Silberstein SD. Preventive treatment of migraine: an overview. Cephalalgia. 1997;17(2):67-72.

14. Silberstein SD. Migraine. Lancet. 2004;363(9406):381-91.

15. Sun L, Zhang S-Q, Zhong D-F. In vitro identification of metabolites of verapamil in rat liver microsomes. Acta Pharmacol Sin. 2004;25:121-8.

16. Ruhé HG, Mason NS, Schene AH. Mood is indirectly related to serotonin, norepinephrine and dopamine levels in humans: a meta-analysis of monoamine depletion studies. Mol Psychiatry. 2007;12(4):331-59.

17. Muscat R, Papp M, Willner P. Antidepressant-like effects of dopamine agonists in an animal model of depression. Biol Psychiatry. 1992;31(9):937-46.

18. Conn P, Sanders-Bush E. Selective 5HT-2 antagonists inhibit serotonin stimulated phosphatidylinositol metabolism in cerebral cortex. Neuropharmacology. 1984;23(8):993-6.

19. Wang C, Jiang Y, Ma J, Wu H, Wacker D, Katritch V, et al. Structural basis for molecular recognition at serotonin receptors. Science. 2013;340(6132):610-4. 